\title{
Relationship of insulin resistance with recurrent pregnancy loss
}

\author{
Asifa Ali Wani ${ }^{1}$, Irfan Gul ${ }^{2 *}$, Farhat Jabeen ${ }^{1}$, Shiveta Kaul ${ }^{1}$, Farhat Ali Lone ${ }^{1}$, Gulshan Akhter ${ }^{1}$
}

\begin{abstract}
${ }^{1}$ Department of Obstetrics and Gynecology, Government Medical College, Srinagar, Jammu and Kashmir, India
${ }^{2}$ Department of Medicine, Government Medical College, Srinagar, Jammu and Kashmir, India
\end{abstract}

Received: 04 March 2017
Accepted: 18 March 2017

\author{
*Correspondence: \\ Dr. Irfan Gul, \\ E-mail: drirfanbeigh@yahoo.in
}

Copyright: (c) the author(s), publisher and licensee Medip Academy. This is an open-access article distributed under the terms of the Creative Commons Attribution Non-Commercial License, which permits unrestricted non-commercial use, distribution, and reproduction in any medium, provided the original work is properly cited.

\section{ABSTRACT}

Background: The recurrent pregnancy loss (RPL) is defined as two and more failed pregnancies as documented by ultrasound and histopathological examination and suggested some assessment after each loss with a thorough evaluation after three or more losses. RPL is one of the most frustrating and difficult areas in reproductive medicine because the aetiology is often unknown and there are few evidence based diagnostic and treatment strategies.

Methods: 150 Non pregnant females were taken as both cases and controls in the study. All the pregnancy losses were documented by ultrasound or histological examination after uterine curettage. The control group consisted of women with no RPL with at least one live birth. These two groups were matched on the basis of age and BMI. All the women underwent following examinations, viz. Hysterosalpingography, karyotype of both partners, serum TSH, FT4, prolactin and antibodies for APLA. In addition blood sample were taken for fasting serum glucose and serum insulin level later insulin resistance was calculate using three parameters Fasting insulin > 20IU/ml. Diagnostic of Insulin Resistance. (2) Fasting glucose / Fasting insulin. A ratio of $<4.5$ being diagnostic of insulin resistance. (3) HOMA IR.

FG (mg/dl) x FPI (IU/ml) FG (mmol/l) x FPI (IU/ml)

$$
\text { OR }
$$

40522.5

Where $1 \mathrm{mmol} / \mathrm{l}=18 \mathrm{mg} / \mathrm{dl}$, A value of $>4.5$ being diagnostic of insulin resistance.

Results: 150 patients were enrolled in this study among which 75 were selected as cases and 75 as controls after fulfilling inclusion and exclusion criteria with mean age cases group was $28.4+2.37$ years and $29.1+2.70$ years in control group mean miscarriage rate in study group was $3.17+83$ and control group with $0.35+0.48$ with statistically significant difference. Mean fasting glucose $(96.5+7.86) \mathrm{mg} / \mathrm{dl}$, Fasting Insulin $(14.1 \pm 5.91) \mathrm{IU} / \mathrm{ml}$. Mean Glucose Insulin ratio $(8.1 \pm 3.39)$, HOMA-IR $(3.4 \pm 1.51)$ in the study group and in control group mean fasting glucose was $(87.1+11.49)$ $\mathrm{mg} / \mathrm{dl}$, Fasting Insulin $(6.9 \pm 4.99) \mathrm{IU} / \mathrm{ml}$. Mean Glucose Insulin ratio $(17.8 \pm 11.44)$, HOMA-IR $(1.5 \pm 1.27)$ respectively with statistically significant difference.

Conclusions: In women with recurrent pregnancy loss fasting insulin and insulin resistance are higher than those in women without spontaneous abortion. The most sensitive parameter for calculating insulin resistance was found to be fasting insulin followed by HOMA - IR and followed by fasting glucose/fasting insulin ratio. It is therefore important to recommend a fasting insulin and fasting glucose level while evaluating a case of recurrent pregnancy loss to assess for insulin resistance.

Keywords: Fasting Insulin, HOMA-IR, Mean fasting glucose, Mean glucose insulin ratio, Re-current pregnancy loss

\section{INTRODUCTION}

Recurrent pregnancy loss (RPL) was traditionally described as three or more clinically diagnosed consecutive pregnancy losses prior to the $20^{\text {th }}$ gestational week but molar and ectopic pregnancies were not included in the definition. ${ }^{1}$ now a day recurrent pregnancy loss is defined as two and more failed pregnancies as documented 
by ultrasound and histopathological examination and suggests some assessment after each loss with a thorough evaluation after three or more losses. ${ }^{2}$ Recurrent pregnancy loss is one of the most difficult areas in reproductive medicine for management because the etiology is often unknown and thus there are few evidence based diagnostic and treatment strategies. ${ }^{3}$

Most of physicians recommend evaluation after the loss of two pregnancies while in developing countries like our will not begin a workup until the loss of at least three pregnancies. ${ }^{4}$ But early testing the woman after two losses could help them of another pregnancy failure and thus lowering the number of spontaneous losses to two. ${ }^{5}$ Recurrent pregnancy loss is estimated to occur in $2-4 \%$ of reproductive age couples. ${ }^{6}$ the incidence of RPL by chance alone would be approximately $0.35 \% .{ }^{7}$ It is a major hazard in pregnancy both after spontaneous conception and after ART treatment. Couples who experience RPL may benefit from a medical evaluation and psychological support. The exact etiology of RPL is not completely understood but the following factors are contributory which include anatomical abnormalities of uterus, endocrine factors, coagulation and immunological causes, infections and chronic diseases, defective placentation, environmental toxins, psychological factors and genetic abnormalities. ${ }^{8}$ Mechanical abnormalities account for only $10-15 \%$ of cases of recurrent pregnancy loss and are usually thought to cause miscarriage by interrupting the vasculature of the endometrium, prompting abnormal and inadequate placentation $^{9}$. Infections such as listeria monocytogenes, toxoplasma gondii, rubella, HSV, measles, CMV and coxsakie viruses are known or suspected to cause sporadic spontaneous pregnancy loss. However the role of infectious agents in recurrent pregnancy loss is not clear with a proposed incidence of 0.5 to $5 \% .{ }^{1}$ Inherited and combined inherited/acquired thrombophilia's are common with more than $15 \%$ of the western population carrying an inherited thrombophilia mutation. ${ }^{10}$ The most common of these are the mutations in factor $\mathrm{V}$ Leiden, prothrombin gene and methylene tetra-hydrofolate reductase. ${ }^{11}$ there is a feeling of concerned about the possibility that environmental factors may have caused their pregnancy losses. ${ }^{12}$ About $2-4 \%$ of RPL is associated with parental balanced structural.

A chromosomal rearrangement is most commonly balanced reciprocal or Robert-Sonian translocations. Other structural abnormalities associated with RPL such chromosomal inversions, insertions and mosaicism. ${ }^{13}$ Diabetes mellitus, thyroid disease, luteal phase deficit, polycystic ovarian syndrome and hyperprolactinemia are most common endocrine disorders causing recurrent pregnancy loss in about $20 \%$. Polycystic ovarian syndrome is most common endocrine disorder present in at least $40 \%$ of women with recurrent pregnancy loss. Insulin resistance and the resultant hyperinsulinemia that is present in metabolic syndrome, type 2 diabetes mellitus and polycystic ovarian syndrome is the major cause of recurrent pregnancy loss as evidenced by the decreased rate of spontaneous pregnancy loss in patients with weight reduction and therapy with the insulin sensitizing drugs particularly metformin. ${ }^{14,15}$ Polycystic ovary syndrome (PCOS)is a complex, heterogenous disorder of uncertain etiology but now a days there are strong evidence that it can be classified as a genetic disease. ${ }^{16-18}$ It is thought to be leading causes of female subfertility and the most frequent endocrine problem in women of reproductive age. $^{19-22}$

There are some reports of high recurrent spontaneous miscarriage rate in overweight, obese, infertile women treated by ART. ${ }^{23}$ High insulin levels have been shown in vitro to increase the transport of glucose by first trimester cytotrophoblasts independent of glucose level (probably by upregulation of GLUT1 glucose transporter system). ${ }^{24}$ The expression of glycodelin and of IGFBP-1 (insulin like growth factor binding protein-1) is decreased by the hyperinsulinemia at the implantation situs. Glycodelin plays an immune role, inhibiting the endometrial response towards the embryo, while the IGFBP-1 facilitates the adhesion process of the blastocyst at the feto-maternal surface. $^{25}$

A second possibility involves PA inhibitor which has been shown to increase with increasing level of insulin and decrease with treatment by an insulin sensitizing agent. ${ }^{26,27}$ It has been suggested that increased PA inhibitor activity promoted recurrent abortion through thrombotic induction of placental insufficiency. ${ }^{28,29}$

The exact mechanism of how insulin resistance leads to RPL is unknown. Insulin resistance and hyperinsulinemia are claimed to be potential causes of high rate of pregnancy loss in patients with PCOS and have been linked to be the metabolic and endocrine abnormalities associated with the pathophysiology of RPL. Insulin resistance is a physiological condition in which cells receptor which is target site for insulin did not respond normal to the action of hormone insulin. ${ }^{30}$ Insulin resistance is often associated with a hypercoagulable state (impaired fibrinolysis) and increased inflammatory cytokine levels. ${ }^{31}$ Insulin resistance has been demonstrated to increase expression of PAI-1. PAI-1 activity is known to elevate levels of serum insulin and it induces a hypo fibrinolytic state. This creates a thrombotic milieu at the materno fetal interface with high risk of miscarriage. ${ }^{3}$

\section{METHODS}

This study was conducted in the Lalla Ded Hospital tertiary care Obstetrics and Gynaecology hospital associated Hospital of Government Medical College, Srinagar conducted over a period of one and a half years (April 2014 to September 2015) after obtaining clearance from Institutional Ethical Committee and proper written consent in local Kashmiri language 140 patient. 


\section{Inclusion criteria}

All patients were in reproductive age group (20-40 years). Patients with history of 2 or more recurrent pregnancy losses. Patients with history of recurrent pregnancy loss in whom other causes of recurrent pregnancy losses were ruled out.

\section{Exclusion criteria}

Patients who were: Diabetic, PCOS, Family history of diabetes, having metabolic syndrome.

About 75 cases and 75 controls fulfilling the inclusion criteria were enrolled for the study after consent. Non pregnant females were taken as both cases and controls in the study. All the pregnancy losses were documented by ultrasound or histological examination after uterine curettage. The control group consisted of women with no RPL with at least one live birth.

These two groups were matched on the basis of age and BMI. All the women underwent following examinations, viz. Hysterosalpingography, karyotype of both partners, serum TSH, FT4, prolactin and antibodies for APLA. All the patients with none of the above factors were included in the case group. The exclusion criteria for both the groups were the presence of diabetes mellitus (the fasting blood glucose level higher than $126 \mathrm{mg} / \mathrm{dl}$, at two different testing levels or the presence of PCOS or family history of diabetes, or having any metabolic syndrome.

PCOS status was determined based on the revised Rotterdam criteria (1) oligo and/or anovulation, (2) clinical and/or biochemical signs of hyperandrogenism). (3) Polycystic ovaries on USG (presence of 12 or more follicles in each ovary measuring $2-9 \mathrm{~mm}$ in diameter and/or increased ovarian volume $>10 \mathrm{ml}$. one ovary fitting this definition is sufficient to define PCOD on USG).

Under all aseptic precautions (UAAP) venous blood samples were drawn after a period of 12 hours fasting from both cases and controls. The blood samples were collected in vials not containing any anticoagulant. The fasting glucose was determined soon after the collection of blood samples, for determining fasting insulin levels, blood samples were spun in centrifuge immediately after collection and serum was separated and kept in separate vials and were stored at about -200C (in refrigerator) for analysis of fasting insulin which was performed later on in the Department of Biochemistry Laboratory, Government Lalla Ded Hospital, Srinagar. Fasting serum insulin was determined by ELISA.

Three parameters were used in this study to calculate insulin resistance. Fasting insulin $>20 \mathrm{IU} / \mathrm{ml}$. (Diagnostic of Insulin Resistance).

\section{HOMA IR}

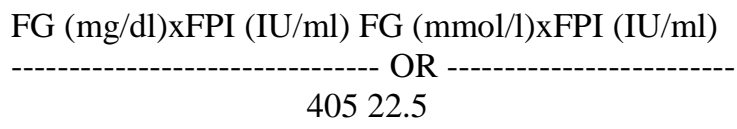

Where $1 \mathrm{mmol} / \mathrm{l}=18 \mathrm{mg} / \mathrm{dl}$

A value of $>4.5$ being diagnostic of insulin resistance.

Fasting glucose / Fasting insulin

A ratio of $<4.5$ being diagnostic of insulin resistance.

\section{RESULTS}

150 patients were enrolled in this study among which 75 were selected as cases and 75 as controls after fulfilling proper inclusion and exclusion criteria. Mean age in the cases group and control group was $28.4 \pm 2.37$ years and $29.1 \pm 2.70$ years respectively with statistically insignificant difference. Mean BMI was also having statistically insignificant difference and mean miscarriage rate in cases group was $3.17+83$ and in control group with $0.35+0.48$ with statistically significant difference.

Table 1: Comparison between two groups based on age.

\begin{tabular}{|c|c|c|c|c|c|}
\hline \multirow{2}{*}{ Age (yrs) } & \multicolumn{2}{|c|}{ Study group } & \multicolumn{2}{|c|}{ Control group } & \multirow{2}{*}{$\begin{array}{l}\text { P- } \\
\text { value }\end{array}$} \\
\hline & No. & $\%$ & No. & $\%$ & \\
\hline $24-26$ & 19 & 25.3 & 16 & 21.3 & \multirow{5}{*}{$\begin{array}{l}0.097 \\
\#\end{array}$} \\
\hline $27-29$ & 34 & 45.3 & 26 & 34.7 & \\
\hline $30-32$ & 18 & 24.0 & 25 & 33.3 & \\
\hline $33-35$ & 4 & 5.3 & 8 & 10.7 & \\
\hline Mean \pm SD & \multicolumn{2}{|c|}{$28.4 \pm 2.37$} & \multicolumn{2}{|c|}{$29.1 \pm 2.70$} & \\
\hline
\end{tabular}

\#Statistically Non Significant Difference (P-value >0.05)

Table 2: Comparison between two groups based of various parameters.

\begin{tabular}{|lll|l|}
\hline Parameters & $\begin{array}{l}\text { Study } \\
\text { group } \\
\text { Mean }\end{array}$ & $\begin{array}{l}\text { Control } \\
\text { group } \\
\text { Mean }\end{array}$ & P value \\
\hline Age (yrs) & $28.4 \pm 2.37$ & $29.1 \pm 2.70$ & 0.097 \\
\hline BMI & $23.9 \pm 2.15$ & $23.5 \pm 2.07$ & 0.302 \\
\hline Miscarriages & $3.17 \pm 0.83$ & $0.35 \pm 0.48$ & $<0.001^{*}$ \\
\hline Fasting glucose & $96.5 \pm 7.86$ & $87.1 \pm 11.49$ & $<0.001^{*}$ \\
\hline Fasting insulin & $14.1 \pm 5.91$ & $6.9 \pm 4.99$ & $<0.001^{*}$ \\
\hline $\begin{array}{l}\text { Glucose; } \\
\text { insulin RATIO }\end{array}$ & $8.1 \pm 3.39$ & $17.8 \pm 11.44$ & $<0.001^{*}$ \\
\hline HOMA-IR & $3.4 \pm 1.51$ & $1.5 \pm 1.27$ & $<0.001^{*}$ \\
\hline
\end{tabular}

*Statistically Significant Difference $(\mathrm{P}$-value $<0.05)$

Mean fasting glucose $(\mathrm{mg} / \mathrm{dl})$ in the cases group was $96.5+7.86$ and in control group was $87.1+11.49$ with statistically significant difference. Mean fasting insulin (mIU/ml) was high in cases group as compared to control group with statistically significant difference. Mean Glucose Insulin ratio in the cases group was 8.1 and in the control group was 17.8. Mean HOMA-IR was 3.4 in the 
cases group and 1.5 in the control group having statistically significant difference.

When insulin resistance was calculated based on fasting insulin it was found to be high in the cases group (26.7\%) as compared to control group (6.7\%).

Table 3: Comparison of insulin resistance in two groups Based on fasting insulin level.

\begin{tabular}{|c|c|c|c|c|c|}
\hline \multirow{2}{*}{$\begin{array}{l}\text { Fasting } \\
\text { insulin } \\
\text { level }\end{array}$} & \multicolumn{2}{|c|}{ Study group } & \multicolumn{2}{|c|}{ Control group } & \multirow[b]{2}{*}{ P-value } \\
\hline & No. & $\%$ & No. & $\%$ & \\
\hline$\geq 20$ & 20 & 26.7 & 5 & 6.7 & \multirow{3}{*}{$0.001 *$} \\
\hline$<20$ & 55 & 73.3 & 70 & 93.3 & \\
\hline Total & 75 & 100 & 75 & 100 & \\
\hline
\end{tabular}

Insulin resistance based on glucose insulin ratio $(<4.5)$ was found to be $13.3 \%$ in study (cases) group as compared to $2.7 \%$ in control group with statistically significant difference.

Table 4: Comparison of insulin resistance in two groups based on glucose insulin ratio.

\begin{tabular}{|c|c|c|c|c|c|}
\hline \multirow{2}{*}{$\begin{array}{l}\text { Glucose } \\
\text { insulin } \\
\text { ratio }\end{array}$} & \multicolumn{2}{|c|}{ Study group } & \multicolumn{2}{|c|}{ Control group } & \multirow{2}{*}{$\begin{array}{l}\text { P- } \\
\text { value }\end{array}$} \\
\hline & No. & $\%$ & No. & $\%$ & \\
\hline$<4.5$ & 10 & 13.3 & 2 & 2.7 & \multirow{3}{*}{$0.035^{*}$} \\
\hline$\geq 4.5$ & 65 & 86.7 & 73 & 97.3 & \\
\hline Total & 75 & 100 & 75 & 100 & \\
\hline
\end{tabular}

Insulin resistance as per HOMA-IR was found to be $24 \%$ in the study (cases) group and $8 \%$ in the control group.

Table 5: Comparison of insulin resistance in two groups based on HOMA-IR.

\begin{tabular}{|c|c|c|c|c|c|}
\hline \multirow{2}{*}{$\begin{array}{l}\text { HOMA- } \\
\text { IR }\end{array}$} & \multicolumn{2}{|c|}{ Study group } & \multicolumn{2}{|c|}{ Control group } & \multirow{2}{*}{ P-value } \\
\hline & No. & $\%$ & No. & $\%$ & \\
\hline$>4.5$ & 18 & 24.0 & 6 & 8.0 & \multirow{3}{*}{$0.003^{*}$} \\
\hline$\leq 4.5$ & 57 & 76.0 & 69 & 92.0 & \\
\hline Total & 75 & 100 & 75 & 100 & \\
\hline
\end{tabular}

*Statistically Significant Difference (P-value <0.05)

In this study three parameters were used to diagnose insulin resistance which were fasting insulin $>20 \mathrm{mIU} / \mathrm{ml}$, HOMA-IR $>4.5$ and FG/FI ratio< 4.5.

Table 6: Insulin resistance by various parameters.

\begin{tabular}{|ll|}
\hline Parameters & $\%$ \\
\hline Fasting insulin $\geq 20$ & 26.7 \\
\hline GI Ratio $<4.5$ & 13.3 \\
\hline HOMA-IR $>4.5$ & 24 \\
\hline
\end{tabular}

\section{DISCUSSION}

In the present study there was no statistical significant difference between two groups with respect to age and BMI. The mean age in the study group was $28.4 \pm 2.37$ years and in control group was $29.1 \pm 2.70$ years with $p$ value of 0.097 which is statistically insignificant. These observations were comparable with the study of Corina Alina et al and Craig LB et al where the mean age in the study group was $30.12 \pm 4.90$ and $32.7 \pm 5.4$ years and in control group was $29.36 \pm 5.7$ years and $32.8 \pm 6.0$ years respectively. ${ }^{25,31}$ The mean BMI in the study group was $23.9 \pm 2.15$ and in the control group was $23.5 \pm 2.07$ with an insignificant $\mathrm{p}$ value of 0.302 . The results obtained were similar to the study of Craig LB et al where mean BMI was $29.2 \pm 7.3$ in the study group and $29.0 \pm 7.2$ in the control group. ${ }^{31}$ These results were also consistent with the study of Celik Nazan et al where the mean BMI in the study group was $25.7 \pm 4.3$ and in the control group was $24.9 \pm 4.2 .^{7}$ In the current study statistically significant difference was noted between two groups with respect to mean miscarriage rate that is $3.17 \pm 0.83$ in the study group and $0.35 \pm 0.48$ in the control group with $p$ value of less than 0.001 which is statistically significant. Similar results were obtained in the study of Celik Nazan et al where the mean miscarriage rate in the study group was $3.0 \pm 1.0$ and control group was $0.2 \pm 0.4 .^{7}$ Another study by Craig LB et al showed mean miscarriage rate of $3.0 \pm 1.2$ in the study group and $0.1 \pm 0.4$ in the control group which were also comparable with the current study. In our study mean fasting glucose in the study group was $96.5 \pm 7.86$ and in the control group was $87.1 \pm 11.49$ with statistically significant $\mathrm{p}$ value $<0.001) .{ }^{31}$ The results were consistent with the study of Celik Nazan et al and KA Wahba et al where mean fasting glucose in the study group was $100.8 \pm$ 12.8 and $86.76 \pm 9.8$ and in control group it was $89.7 \pm 15.1$ and $84.44 \pm 9.12$ respectively. ${ }^{7,32}$ The mean fasting insulin in the study group was $14.1 \pm 5.91$ and in control group it was $6.9 \pm 4.99$ with $p$ value of $<0.001$ with a statistically significant difference. Studies conducted by Wang Y Zhao et al, Kotanaie Maryam et al and KA Wahba et al observed similar results. ${ }^{6,32,35}$ It was noted that the mean glucose insulin ratio in the study group was $8.1 \pm 3.39$ and in control group was $17.8 \pm 11.44$, while comparing the two groups statistically significant difference was found with a value of $0<.001$. Similar inferences were made by Celik Nazan et al where the mean glucose insulin ratio in the study group was $12.2 \pm 13.5$ and in the control group was $28.3+15.4$ with statistically significant difference ( $\mathrm{p}$ value $<0.001){ }^{7}$ Study done by Li ZL et al and KA Wahba (2015) also observed significant difference with a $\mathrm{p}$ value $<0.001$ with respect to mean GI ratio in the study and control group. ${ }^{32,33}$ In the present study the mean HOMA-IR in the study group was $3.4 \pm 1.51$ and in the control group was $1.5 \pm 1.27$. The difference was statistically significant with a $\mathrm{p}$ value of $<0.001$. This was proved by several authors in their study like Corina-Alina et al where mean HOMAIR in the study group was 2.98 and in the control group was 1.69 with a significant $\mathrm{p}$ value $<0.001$ and Celik Nazan et al where mean HOMA-IR in the study group was 
4.16 and in the control group was 1.62 having statistically significant difference $(p<0.001) .^{7,25}$ Insulin resistance which was measured in terms of fasting insulin (>20mIU/ml) was present in $26.7 \%$ of patients in the study group and $6.7 \%$ patients in the control group. The difference was statistically significant with a $\mathrm{p}$ value of $<0.006$. These results were consistent with the study conducted by Craig LB et al where insulin resistance was observed in $25.7 \%$ of patients in study group and $8.1 \%$ of patients in the control group with a statistically significant difference ( $p$ value of $<0.001$ ). Similar results were observed by Corona Alina et al. ${ }^{25,31}$ In the current study insulin resistance based on glucose insulin ratio (<4.5) was observed in $13.3 \%$ of the patients in the study group and $2.7 \%$ patients in the control group with a statistically significant different $(<0.035)$. Our results were comparable with the study conducted by Craig LB et al and Li ZL et al with statistically significant difference ( $p$ $<0.001)$. Insulin resistance measured by HOMA-IR $(>4.5)$ was observed in $24 \%$ of patients in the study group and $8 \%$ of patients in the control group with statistically significant difference $(p<0.008){ }^{31,33}$ Similar observations were made by Corona Alina et al and Michael Diejomaoh et al with statistically significant difference $(p<0.001) .^{25,34}$

\section{CONCLUSSION}

From the present study and the results, it was concluded that:

- In women with recurrent pregnancy loss fasting insulin and insulin resistance are higher than those in women without spontaneous abortion.

- Recurrent pregnancy losses are associated with insulin resistance.

- The most sensitive parameter for calculating insulin resistance was found to be fasting insulin followed by HOMA - IR and followed by fasting glucose/fasting insulin ratio.

It is therefore important to recommend a fasting insulin and fasting glucose level while evaluating a case of recurrent pregnancy loss to assess for insulin resistance as it may require treatment which includes life style changes, exercise and insulin sensitizing drugs like metformin.

\section{Funding: No funding sources}

Conflict of interest: None declared

Ethical approval: The study was approved by the Institutional Ethics Committee

\section{REFERENCES}

1. Fox Lee Laura, Schust DJ. RPL in Berek, Jsed and Novak Gynaecology 14th Ed. Phillipedia, Lippincott Williams and Wilkins. 2007;1277-322.

2. Practice Committee of the American Society for Reproductive Medicine. Definitions of infertility and recurrent pregnancy loss. Fertil Steril 2008;89(6):1603.
3. Rayah S. Baban A. Al-Moayed. Serum leptin and insulin hormone level in recurrent pregnancy loss. Oman Med J. 2010;25(3):203-07.

4. Grattan D, Ladyman S. Hormonal induction of leptin resistance during pregnancy. Physiology and Behaviour. 2007;91(4):366-74.

5. Practice Committee of American Society for Reproductive Medicine. Definitions of infertility and recurrent pregnancy loss. Fertility and Sterility 2008;90:60.

6. Kotaniae Maryam. The comparison of insulin resistance frequency in patients with recurrent early pregnancy loss to normal individuals. BMC Research Notes 2012;133.

7. Celik Nazan. Evaluation of the relationship between insulin resistance and recurrent pregnancy loss. Ginekologia Polska. 2011;82:272-75.

8. Sudha Salhan. Recurrent pregnancy loss. In: Textbook of Gynaecology. 1st Edition. 2011;184-88.

9. Lin PC. Reproductive outcome in women with uterine anomalies. Women Health. 2004;13:36-9.

10. Green IA, Thrombophilia: complication for pregnancy outcome. Thromb Res 2003; 109: 73-81.

11. Lane DA and Grat P. Role of hemostatin gene polymorphism in women. Thromphia. 2000;95:151732.

12. Management of RPL. Washington DC. The American College of Obstetrics and Gynaecology the ACOG Practice Bulletin No. 2011;24.

13. Stephenson MD. Frequency of factors associated with habitual abortion in 197 couples. Fertil Steril. 1996;66:24-9.

14. Ford HB, Schust DJ. Recurrent pregnancy loss: etiology, diagnosis, and therapy. Rev Obstet Gynecol. 2009;2(2):76-83.

15. Kassie J.et al Genetic considerations in RPL. Cold Spring Harb Perspect Med 2015; 5: a023119.

16. Fauser BCGM. Contemporary genetic technologics and female reproduction. Humans Reproduction Update. 2011;17(6):829-47.

17. Legro RS; Strauss JF. Molecular progress in infertility: polycystic ovary syndrome. Fertility and Sterility Sept. 2002;78(3):569-76.

18. Diamanti-Kandarakis. The role of genes and environment in the etiology of PCOS. Endocrine. 2006;30(1):19-26.

19. Goldenberg. Medical therapy in women with polycystic ovary syndrome before and during pregnancy and lactation. Minerva Ginecol. 2008;60(1):63-75.

20. Boomsma CM. Pregnancy complications in women with polycystic ovary syndrome. Semin. Reprod. Med. 2008;26(1):72-84.

21. Azziz R. The Prevalence and Features of the Polycystic Ovary Syndrome in an Unselected Population. Journal of Clinical Endocrinology and Metabolism. 2004;89(6):2745-9.

22. H Teede, A. Polycystic ovary syndrome: a complex condition with psychological, reproductive and 
metabolic manifestations that impacts on health across the lifespan. BMC Medicine J. 2010;8:41.

23. Wang JX. Obesity increases the risk of spontaneous abortion during infertility treatment. Obes Res. 2002;10:551-4.

24. Gordon MC, Zimmerman PD. Insulin and glucose modulate glucose messenger ribonucleic acid expression and glucose uptake in trophoblasts isolated from first-trimester chorionic villi. Am J Obstet Gynecol. 1995;173:189-97.

25. Corrina Alina, IS Pasou. High fasting insulin levels and insulin resistance may be linked to idiopathic RPL. Journal of Endocrinology. 2013;3.

26. Glueck CJ, Wang. Plasminogen activator inhibitor activity: an independent risk factor for the high miscarriage rate during pregnancy in women with polycystic ovarian syndrome. Metabolism. 1999;48: 1589-95.

27. Glueck CJ, Phillips H. Continuing metformin throughout pregnancy in women with polycystic ovarian syndrome appears to safely reduce firsttrimester abortion: a pilot study. Fertil Teril. 2001;75:46-52.

28. Gris JC, Ripart Neeu S. Respective evaluation of the prevalence of haemostasis abnormalities in unexplained primary early recurrent miscarriages. The Nimes Obstetricians and Haematologists (NOHA) Study. Thromb Haemost. 1997;77:1096103.
29. Gris JC, Neveu S. Plasma fibrinolytic activators and their inhibitors in women suffering from early recurrent abortion of unknown etiology. J Lab Clin Med. 1993;122:606-15.

30. DJ Jakubowicz, PA Essah. Reduced serum glycodelin and insulin like growth factor binding protein-1 in women with polycystic ovary syndrome during first trimester of pregnancy. Journal of Clinical Endocrinology and Metabolism 2004;89(2):833-39.

31. Craig LB, Ke RW. Increased prevalence of insulin resistance in women with a history of recurrent pregnancy loss. Fertil Steril. 2002;78(3):487-90.

32. K.A. Wahba. Assessment of the Role of Insulin Resistance in Cases of Recurrent Unexplained First Trimesteric Miscarriage. Life Science Journal 2015;12(7):174-81.

33. Li ZL, Xiang HF. Association between recurrent miscarriages and insulin resistance: a meta-analysis. Zhonghua Fu Chan Ke Za Zhi. 2012;47(12):915-9.

34. Michael Diejomaoh. Insulin resistance in women with recurrent spontaneous miscarriage of unknown etiology. Med Princ Pract. 2007;16:114-18.

35. Wang Y, Zhao H. Relationship between recurrent miscarriage and insulin resistance. Gynecol Obstet Invest. 2011;72(4):245-51.

Cite this article as: Wani AA, Gul I, Jabeen F, Kaul S, Lone FA, Akhter G. Relationship of insulin resistance with recurrent pregnancy loss. Int J Reprod Contracept Obstet Gynecol 2017;6:1312-7. 